\title{
Barriers and Facilitators of Adherence to Anti-Diabetic Therapy in Pregnant Women with Diabetes: Health Care Workers' Perspectives
}

\author{
Doreen Macherera Mukona1, Stephen Peter Munjanja², Mathilda Zvinavashe1, \\ Babill Stray-Pederson ${ }^{3}$ \\ ${ }^{1}$ Department of Nursing Science, University of Zimbabwe College of Health Sciences, Harare, Zimbabwe \\ ${ }^{2}$ Department of Obstetrics and Gynaecology, University of Zimbabwe College of Health Sciences, Harare, Zimbabwe \\ ${ }^{3}$ Institute of Clinical Medicine, Oslo University and Division Women and Children Rikshospitalet Oslo University Hospital, Oslo, \\ Norway \\ Email: dmacherera@yahoo.co.uk, zvinava@yahoo.com,spmunjanja@gmail.com, babill.stray-pedersen@medisin.uio.no
}

How to cite this paper: Mukona, D.M., Munjanja, S.P., Zvinavashe, M. and StrayPederson, B. (2017) Barriers and Facilitators of Adherence to Anti-Diabetic Therapy in Pregnant Women with Diabetes: Health Care Workers' Perspectives. Journal of Diabetes Mellitus, 7, 160-174.

https://doi.org/10.4236/jdm.2017.73013

Received: May 21, 2017

Accepted: August 13, 2017

Published: August 16, 2017

Copyright () 2017 by authors and Scientific Research Publishing Inc. This work is licensed under the Creative Commons Attribution International License (CC BY 4.0).

http://creativecommons.org/licenses/by/4.0/

\begin{abstract}
Global prevalence of hyperglycaemia in pregnancy in women of 20 - 49 years was estimated to be $16.9 \%$ and affecting 21.4 million live births, in $2013,90 \%$ of which occurred in developing countries. The cornerstone of anti-diabetic therapy is diet, physical activity and medications. The study utilized a qualitative descriptive design using key informant interviews from August 2016 to November 2016 to explore challenges of adherence to anti-diabetic therapy in pregnant women with diabetes at a central hospital in Harare, Zimbabwe. Permission to conduct the study was obtained from the respective local and national ethical review boards. All participants gave verbal and written informed consent. A sample of eight key informants directly involved in the care of pregnant women with diabetes was purposively selected for key informant interviews. Key informants should have worked with diabetic pregnant women for at least one year. Sample size was determined by data saturation. Interviews followed a semi structured questionnaire that had sections on the burden of diabetes in pregnancy, challenges of adherence, challenges in management and possible solutions to challenges faced. All interviews were conducted in a private room. Detailed notes were taken during the interviews which were also being audiotaped. Trustworthiness was achieved by observing credibility, dependability, transferability and confirmability. Thematic analysis was done. Thematic analysis was done manually. The stages of data analysis followed were data organization, familiarization, transcription, coding, developing a thematic framework, indexing, displaying and reporting. Major themes identified were barriers and facilitators of adherence to anti-diabetic therapy. Categories under barriers were financial barriers, lack of health edu-
\end{abstract}


cation, lack of trained personnel, shortage of staff and lack of collaboration among practitioners. Categories under facilitators of adherence were subsidization of care, formal training of professionals, promoting collaboration and establishment of a unit dedicated to the care of pregnant women with diabetes. Barriers and solutions identified should be utilized to develop frameworks to promote adherence to anti-diabetic therapy incidence of adverse perinatal outcomes.

\section{Keywords}

Diabetes in Pregnancy, Adherence, Perinatal Outcomes, Health Care Workers

\section{Introduction}

Diabetes mellitus in pregnancy can be pregestational or gestational diabetes [1]. Pregestational diabetes is Type I or Type II DM that existed before conception (Fong et al., 2014) while gestational diabetes (GDM) is glucose intolerance first detected during pregnancy [2]. However both are characterized by glucose intolerance though both pregestational type 1 diabetes and type 2 diabetes confer significantly greater risk than GDM [3].

Urbanization in Africa, has largely contributed to the evolving problem of chronic diseases, mainly diabetes mellitus [4]. This rise will most likely result in an increase in diabetes in pregnancy in Africa, though the prevalence of GDM is relatively unknown [5]. The incidence of GDM reflects the incidence of type 2 diabetes mellitus in the background population [6].

Global prevalence of hyperglycaemia in pregnancy in women of $20-49$ years was estimated to be $16.9 \%$ and affecting 21.4 million live births, in $2013,90 \%$ of which are estimated to occur in low- and middle-income countries [4]. The prevalence varies depending on the population and diagnostic criteria used. A prevalence of $0 \%$ to $9 \%$ based on five studies in Sub-Saharan Africa has been reported [7]. Another review of 14 African countries reported a prevalence range from $0 \%$ in Tanzania to $13.9 \%$ in high-risk women in Nigeria [5]. Prevalence rates of GDM range between $6 \%$ and $14 \%$ in East and West Africa [8] and between 13\% and 18\% in South Asia [9]. Approximately 700,000 women give birth in England and Wales each year, about 5\% of which have either pre-existing diabetes or gestational diabetes. Of women who have diabetes during pregnancy, it is estimated that approximately $87.5 \%$ have gestational diabetes $7.5 \%$ have type 1 diabetes and the remaining $5 \%$ have type 2 diabetes [10].

GDM screening differs from country to country and even within countries [5], but in developing countries it is not performed routinely [11] Universal screening is not always feasible from a cost perspective, particularly in low- or middle-income countries (LMICs). Even some high-income countries employ selective screening [12]. Overreliance on adequate nutrition and a fear of administering insulin by health care workers based on fear of hypoglycaemia are oth- 
er reasons for poorly controlled diabetes during pregnancy [13].

Screening for and treating diabetes in pregnancy lead to the reduction of perinatal morbidity and the improvement of post-delivery outcomes [14]. Considering the limited timeframe for management from detection until birth, timely and adequate management of patients is pertinent to reduce potential complications [11]. Diabetes in pregnancy is associated with pregnancy-induced hypertension, pre-eclampsia, antepartum haemorrhage, caesarean, preterm birth, birth trauma and congenital anomalies [15].

The cornerstone of diabetes management is glycemic control which leads to reduction in adverse outcomes in pregnant women with diabetes. The three cornerstones of diabetes management are diet, physical activity, and medication, if necessary [16]. Nutritional therapy is an integral component in the prevention and management of diabetes mellitus and has been shown to result in sustained improvements in glycated hemoglobin (HbA1c) in clinical trials [17].

Adherence to healthy eating and exercise to maintain healthy weight during pregnancy remains problematic [18]. It is important to identify and address individual psychological aspects and the impact they may have on successful behavioral change in pregnant women [19]. Management of pregnant women with diabetes in pregnancy therefore is a challenge that health care professionals face daily because of the complexity of the therapeutic regimen for gestational diabetes mellitus [20]. Management of GDM is a perfect window of opportunity for the prevention of diabetes mellitus in two generations, and awareness of the condition among antenatal women will translate into prevention and early diagnosis of the disease [21]. It is important to understand challenges in the management of diabetes in pregnancy in low resource settings considering the challenges of access to care and resource availability [11]. Employing practitioners' perceptions about the motivators and barriers for women with diabetes to achieve and maintain glycemic control perinatally should be part of the equation for developing approaches to improve pregnancy and long-term outcomes for diabetic women [22].

\section{Purpose}

The purpose of this article is to highlight the health care worker's perspective of barriers and facilitators of adherence to anti-diabetic therapy in pregnant women with diabetes.

\section{Methods}

The study utilised a qualitative descriptive design using key informant interviews from August 2016 to November 2016 to explore challenges of adherence to antidiabetic therapy in pregnant women with diabetes at a central hospital in $\mathrm{Ha}$ rare, Zimbabwe. Permission to conduct the study was obtained from the respective local and national ethical review boards. All participants gave verbal and written informed consent. A sample of eight key informants directly involved in the care of pregnant women with diabetes was purposively selected for key in- 
formant interviews. They included obstetricians, physicians, midwives and dieticians. Key informants must have worked with pregnant women with diabetes for at least a year post qualification. Sample size was determined by data saturation. Data saturation may be attained by as little as six interviews [23]. Interviews were conducted in a private room. Detailed notes were taken during the interviews which were also being audiotaped. Interviews followed a semi structured questionnaire.

Trustworthiness was achieved by credibility, transferability, confirmability and dependability of data collection and analysis. Credibility and dependability were ensured by prolonged engagement, persistent observation, triangulation, peer debriefing, negative case analysis, and member-checking. Transferability was achieved by thick description of the process adopted for the research for others to follow and replicate. Confirmability was achieved by member and data audit which examined any potential biases during data collection and analysis.

Aspects of thematic analysis as proposed by Braun and Clarke [24], Miles and Huberman [25] and Miles et al. [26] were employed in data analysis. Thematic analysis was done manually. The stages of data analysis followed were data organization, familiarization, transcription, coding, developing a thematic framework (searching for themes, reviewing, defining and naming them), indexing, displaying and reporting (interrelating and description of themes). Data was presented as themes.

\section{Findings}

Table 1 presents demographic data of the participants. There were 6 females and 2 males and the sample comprised dieticians, midwives, and medical doctors. They had all attained tertiary education and all had more than 5 years' experience.

Two major themes, namely barriers to adherence and facilitators of management of diabetes in pregnancy were identified. Table 2 presents results of the themes and the categories identified during analysis.

\subsection{Barriers to Adherence to Anti-Diabetic Therapy}

\section{Financial barriers}

All participants mentioned lack of finances as a barrier to adherence to anti-

Table 1. Demographic characteristics for participants for KII $(\mathrm{N}=8)$.

\begin{tabular}{ccccccccc}
\hline & KI 1 & KI 2 & KI 3 & KI 4 & KI 5 & KI 6 & KI 7 & KI 8 \\
\hline AR & $20-30$ & $30-40$ & $30-40$ & $60-70$ & $30-40$ & $30-40$ & $60-70$ & $50-60$ \\
Gender & F & F & F & M & F & F & F & M \\
LOE & T & T & T & T & T & T & T & T \\
Profession & D & M & M & MD & D & MD & M & MD \\
YOE & 6 & 9 & 8 & 42 & 9 & 12 & 33 & 27 \\
\hline
\end{tabular}

Key KI1-KI8-Key informant 1 to Key informant 8; AR-Age range; LOE-Level of education; YOE-Years of experience; F-Female; M-Male; T-Tertiary; D-Dietician; M-Midwife; MD-Medical doctor. 
Table 2. Barriers and facilitators of management of diabetes in pregnancy.

\begin{tabular}{|c|c|c|}
\hline & Barriers to Management & Facilitators of Management \\
\hline Categories & $\begin{array}{l}\text { a. Financial barriers } \\
\text { b. Lack of health education on diabetes } \\
\text { in pregnancy } \\
\text { c. Lack of trained personnel } \\
\text { d. Lack of collaboration among health } \\
\text { care workers } \\
\text { e. Shortage of staff }\end{array}$ & $\begin{array}{l}\text { a. Subsidization of care } \\
\text { b. Strengthening health education } \\
\text { c. Formal training of health care workers } \\
\text { in management of diabetes in pregnancy } \\
\text { d. Collaboration among health care } \\
\text { workers } \\
\text { e. Establishment of a unit dedicated to care } \\
\text { of pregnant women with diabetes }\end{array}$ \\
\hline
\end{tabular}

diabetic therapy. Participants mentioned that there was lack of finances to buy equipment needed in the care of pregnant diabetics such as glucometers. There are no up-to-date drugs being used such as insulin analogues and insulin pumps especially for patients with type 1 diabetes. There are no finances to conduct investigations. The hospital sometimes fails to acquire glucose testing strips, medications and even enough food for the patients who are admitted. This is indicated in the following excerpts:

"The major barrier to adherence is lack of finances, on the part of the patients and the hospital itself. There are no up to date drugs in the hospitals such as insulin analogues that have milder side effects than human insulin. These are likely to promote adherence in patients. There are tests that are never done in our setting such as the fructosamine test that measures glycated albumin. It is more sensitive in pregnancy and gives a more accurate measure of blood glucose control. Patients fail to have their blood glucose checked at the hospital and it is even worse when they are alone at home. Monitoring of blood glucose is an important component of adherence as it directs the type of treatment needed together with diet and exercise requirements." (Male, 63)

"Lack of finances by the hospital itself also affects adherence of patients in a negative way. Admitted patients sometimes fail to get their snacks which are an important part of their diet. The four hourly blood sugar checks together with pre and post prandial checks are not done due to lack of glucose testing strips and sometimes lack of functioning glucometers. This directly affects adherence negatively." (Female, 31)

\section{* Lack of health education on diabetes in pregnancy}

Participants mentioned that there was no proper health education being given to women with diabetes in pregnancy. Diabetes in pregnancy is not viewed as a separate form of diabetes so there is proper education given. Health education on diabetes in pregnancy, its signs and symptoms and the drugs used is vital in enhancing adherence to therapy. The pregnant women are not taught about selfmonitoring of blood glucose, when to and when not to inject insulin. In ANC diabetics are not treated separately from other pregnant women and the health education given there is not specific to diabetes in pregnancy. Physical activity is never talked about and the physiotherapist is not involved in care. Families and communities are also not aware of diabetes in pregnancy and its fundamental differences from diabetes in a non-pregnant individual. As a result patients do 
not have the correct knowledge practices and attitudes towards diabetes in pregnancy. Lack of health education results in bad practices such as fasting during pregnancy, use of herbs and embarking on fad diets. Consultation of traditional and faith healers that often discourage the use of conventional medicines is another result of lack of education. Lack of education also results in late booking. Pregnancy practices such as eating for two, poor food choices and aversion for certain health foods can be addressed by giving health education.

"Health education given to patients is not enough. They are treated like any other patient in ANC so there is no specific health education for diabetes in pregnancy. There is no individualized health education to suit specific needs of different patients. As for diet, there is a universal diabetic diet for patients instead of an individualized one that takes into account unique patient needs. If patients are well educated they are empowered to take control of their condition. Physical activity is not taught as well." (Female, 38)

"Health education is lacking on diabetes in pregnancy because the personnel generally are not well trained to look after pregnant diabetics. When you see these women going to traditional healers or embarking on herbs, it is basically the lack of understanding of their condition and how it is effectively managed. This can be addressed by giving comprehensive health education. Even the excuses of nausea and vomiting and lack of appetite can be addressed by proper individualized health education. This is lacking in our setting due to congestion in the ANC coupled with staff shortages and lack of knowledge on the part of staff.' (Male, 52)

\section{* Lack of trained personnel}

Participants mentioned lack of trained personnel. They mentioned that there was need to train personnel in the management of diabetes in pregnancy. Some participants felt that they were not adequately prepared to care for women with diabetes in pregnancy. One participant mentioned that some specialists were not available in the setting such as endocrinologists, podiatrists and diabetes educators. This is represented by the following excerpts:

"There is lack of trained personnel to manage women with diabetes in pregnancy. In our setting there is only one diabetologist. There are no endocrinologists, podiatrists and diabetes educators. There should be a specialist in every facet of diabetes care and this is not the case in our setting. This negatively impacts adherence as available staff might not be competent to give certain aspects of care let alone the health education required." (Male, 52)

"Staff needs to be trained in order for them to be knowledgeable enough to offer effective health education to patients. Knowledge of one's conditions helps to make them adhere to therapy. In service trainings in diabetes in pregnancy can be done to equip staff with up to date knowledge that benefits patients." (Female, 29)

\section{* Lack of collaboration among health care professionals}

All participants cited that collaboration was lacking among the health care professionals. Management is mainly done by the obstetrician and gynaecolo- 
gists and the midwives. There is need to collaborate with other professionals such as physiotherapists, counsellors and social workers. One participant mentioned that the dietician is only involved when there is a new diabetic and when the blood sugar is difficult to control. There is need for collaboration in all facets of care for diabetes in pregnancy.

"There is overdependence on the doctor for care of these women. Other professionals are not quite involved and this will have negative effects on the patients given the multifaceted nature of diabetes in pregnancy. There is need to collaborate with physiotherapists, counsellors, social workers and even utilize peer support among the patients themselves. This ensures that every aspect of management is addressed including adherence to therapy." (Female, 29)

"We must adopt a multi-disciplinary approach in the care of women with diabetes. This ensures that most if not all the needs of our patients are met. Pregnant women with diabetes have financial, psychological and even spiritual problems besides the diabetes. All these need to be addressed if we are to effectively manage the diabetes." (Female, 38)

\section{* Shortage of staff}

There is a shortage of staff in the ANC and on the wards that was mentioned by all the participants. There is a shortage of midwives, nurses and even the doctors looking after the patients. As a result of the shortage of staff there is not enough time for health education of these women. Psychosocial assessment is not done and this reduces the quality of care.

"There is shortage of staff in the ANC. When these women come for their check-ups, blood sugar is sometimes not checked, individualized health education and psychosocial assessment are not done. If a patient is having problems adhering to therapy it means their issues are not addressed because of shortages." (Female, 31)

"There is a lot of work to be done in ANC by only few midwives and nurses. The pregnant diabetics end up being treated like anyone else because there is no time to give them individualized care. We must weigh the patients when they come, check their blood pressures, take their histories and do the palpations. Besides we also have hypertensive patients and HIV positive pregnant women that we will be taking care of at the same time. This greatly compromises the quality of care we give to the pregnant diabetics." (Female, 29)

\subsection{Facilitators of Adherence to Anti-Diabetic Therapy}

\section{* Subsidization of costs of care}

All participants mentioned the importance of subsidizing care as a facilitator of adherence to anti-diabetic therapy. Participants mentioned the subsidization of costs of medications, syringes, glucose testing strips and investigations so they are affordable to patients. Some suggested free admission as there are usually more than one and lengthy admissions when someone is pregnant.

"Insulin is expensive. There are tests required when one is pregnant such as HBA1Cand many scans. All these require money which the patients do not have. 
Most of our patients here are of socio economic status. Patients end up not adhering to treatment due to lack of funds. If they could get the insulin and glucose testing strips at a lower cost this would help promote adherence. When they get admitted it is even worse because admission costs USD $\$ 12$ for the bed only without the medications. If admission could be made free this would ease the financial burden. As a result most women do not book early for fear of these lengthy admissions yet early booking is essential for strict glycemic control." (Female, 64)

"The hospital should play its part in fostering adherence to anti-diabetic therapy by offering some of the services required during pregnancy for free. There should collaboration with the ZDA for acquisition of insulin, and glucose testing strips. The level of blood sugar determines the dose of insulin or oral medications to be taken. Giving medications without first testing for blood sugar is also a form of non-adherence to therapy. Scans and other specialized tests required should be heavily subsidized so that patients have access to them." (Female, 29)

\section{* Strengthening health education}

All participants mentioned that health education facilitates adherence to antidiabetic therapy. Some of the emerging themes were education about diabetes in pregnancy to enhance understanding of the need for stricter blood glucose control in pregnancy, knowledge of signs and symptoms of complications, and knowledge of the importance of adherence to therapy.

"It is very important to give health education to women with diabetes in pregnancy. This not only empowers them to take control and initiative in their care but it also fosters cooperation from them. If they are made to understand that stricter blood sugar control is required in pregnancy for the safety of the baby they will adhere to therapy in spite of the pica, nausea and vomiting and other problems they have in pregnancy." (Female, 31)

"Health education is very essential in fostering adherence to therapy. Health education should extend to families, communities and the nation at large. This will address problems such as dependence on traditional healers and use of herbs in the management of diabetes in pregnancy that many times will result in complications of diabetes. It will reduce the concurrent use of herbs and conventional medicines that can reduce the efficacy of conventional medicines. Women should be taught about the importance of early booking, during the first trimester, so that blood glucose control starts early in pregnancy." (Male, 52)

\section{* Formal training of health care workers in management of diabetes in pregnancy}

All participants mentioned formal training of health care professionals in the management of diabetes in pregnancy as a facilitator of adherence to anti-diabetic therapy. Five out of the eight key informants suggested a formal qualification for health care professionals while 3 just highlighted that training was important. Three mentioned formal training of nurses and midwives, one mentioned training of diabetes educators while one suggested training of podiatrists and endocrinologist. This is highlighted in the following excerpts: 
"There should be deliberate policy to drive for education of the health care workers. Diabetes in pregnancy is not well understood and health care workers might not be prepared to give proper health education. This directly affects adherence of patients to therapy. They end up consulting traditional healers who claim to be doing everything good for the patient. We need to train endocrinologists, podiatrists, diabetes educators and other professionals if we are to offer comprehensive care to our patients. (Male, 52)

"In service training is important for nurses, midwives and all other health care professionals involved. We do have a protocol for care of diabetes in pregnancy in maternity but it is not followed. If staff are trained in the care and fully understand the rationale behind the protocol they will make an effort to follow the protocol. We should complement the patient's efforts to adhere to therapy. For example we should make sure that admitted patients have their blood sugar checked 4 hourly and they should have their snacks on time. If the hospital does not have strips or snacks we should notify the relatives in time. Of course some relatives won't afford as well but in some cases relatives buy for the patient. (Female, 64)

\section{* Strengthening collaboration among health care workers}

All participants mentioned the strengthening of collaboration among health care workers involved in the care of women with diabetes in pregnancy. They mentioned collaboration among nurses, midwives, dieticians, physicians, obstetricians and gynaecologists, ophthalmologists, counsellors and social workers. These specialists are all available in government hospitals.

"There should be strong collaboration among health care professionals not to leave all pregnant women to the maternity department. This ensures that pregnant women receive adequate care and health education about lifestyle modification especially in pregnancy. Social workers might also help address financial needs of the patients. Collaboration also relieves the congestion in maternity and ensures that patients receive comprehensive care."(Female, 64)

"Care of women with diabetes should involve other specialists. Physicians from the diabetic clinic should continue seeing these patients in maternity. They should be seen by ophthalmologists because they also have eye problems. The dietician should see the patients routinely not just as a referral to enhance adherence to diet. The physiotherapist should also see the patients and really teach them which exercises to do in pregnancy because they do not know them. We can improve adherence by improving our collaboration as health care workers without the patient necessarily needing to pay more money." (Female, 32)

"Collaboration is very necessary in the management of diabetes in pregnancy. There are a lot of factors to be considered not only changing them from oral anti-diabetic drugs to insulin. If they are hypertensive as well their anti-hypertensive drugs need to be changed. There should also be pre conceptual care for women with pre-existing diabetes because they should be taking contraception that is safe for someone with diabetes." (Male, 52) 
Establishment of a unit dedicated to care of pregnant women with diabetes

Some participants mentioned the importance of setting up a unit dedicated to care of women with diabetes in pregnancy. This will help in offering comprehensive care including pre conceptual care. This will enhance collaboration among health care professionals that will in turn help to enhance adherence of pregnant women to anti-diabetic therapy. This is shown in the following excerpts:

"It is very important to set up a unit dedicated to the care of pregnant women with diabetics. This is important to cater for their unique needs for stricter blood sugar control. This has worked well in the care of people living with HIV and AIDS. The unit should be manned by all health care specialists involved in the care. Seeing them in our ANC here results in lack of proper health education and inadequate attention given to them." (Female, 38)

"One way of improving adherence in pregnant women with diabetes in pregnancy is to see them on their own. This will ensure individualized care for them. $A$ unit can be set aside for the care of pregnant diabetics manned by all the health care professionals involved. This ensures comprehensive care."(Male, 52)

\section{Discussion}

The study identified 5 categories under barriers to management of diabetes in pregnancy. However, the barriers identified have been identified elsewhere in other studies.

\section{* Financial barriers}

Limited finances are an obstacle to both diabetes treatment adherence and regular prenatal care [11] [22] [27] [28]. Another review identified financial barriers as hampering proper diabetes in pregnancy management [11]. They however cited lack of accessibility to regular follow up and glucose self-testing. It also came out in this study that equipment for self-testing was not always available in the hospital worse still for patients at home. Another also identified lack of access to care as a barrier to management being largely influenced by financial constraints [22]. The site at which the study was conducted is a government public institution and most patients that come there will not be on any medical insurance. There is need for referral of such patients to the social worker [27] considering the financial burden that diabetes in pregnancy imposes on the patient and family. Addressing a patient's financial barriers builds rapport with her and demonstrates understanding of the health care provider of what she will be facing [27].

\section{* Lack of health education}

Challenges in delivering gestational diabetes care and education in low-resource settings include among other challenges the potential for lower literacy and health literacy levels [29]. Lack of health education was also cited under barriers to adherence to anti-diabetic therapy. It was cited by some key informants that other barriers such as effects of pregnancy and lack of social support could be addressed by effective health education of the pregnant woman and her fami- 
ly. Patient's knowledge gap about the disease pathophysiology, ways to control GDM, and possible adverse outcomes for mother and fetus is a major barrier to diabetes control [27]. Emphasize the importance of preventing adverse outcomes of uncontrolled blood sugar, making necessary lifestyle changes and adhering to the treatment regimen to reduce or prevent complications and improve outcomes [27]. Health education for women with diabetes should focus on pathophysiology of GDM, performing SMBG and explaining the need for strict diet adherence with the ultimate goal of controlling blood glucose, allowing for appropriate weight gain, and avoiding complications for both mother and baby [29].

\section{* Shortage of staff and lack of trained staff}

Shortage of trained health care providers is a main barrier within the health system [28]. There are not enough health care providers to take care of the patient load and also not enough health care providers with adequate training to provide quality care [28]. Some participants in this study mentioned shortage of staff trained in the care of pregnant women with diabetes. These included diabetes nurse educators. There is need to offer specialized training in diabetes in pregnancy in order to improve care and reduce adverse perinatal outcomes. In a study conducted by Mersereau et al. practitioners seemed to consider barriers of access to care and attitude barriers to be beyond their locus of control and did not address them [22]. Proper training in the care of pregnant women with diabetes can equip practitioners with the necessary skills to improve attitudes to care as well as access. These might include effective utilization of proper referral systems and lobbying for financial support for women with diabetes in pregnancy. Participants also mentioned absolute lack of staff and this affected the quality of care especially health education given to the pregnant women. Helping practitioners know what and how to address the needs of childbearing women with or at risk for diabetes can be beneficial [22].

\section{* Lack of collaboration}

Effective collaboration is also necessary in the care of women with diabetes in pregnancy. There is need to appreciate the multifaceted nature of diabetes in pregnancy. There is need for multidisciplinary healthcare teams need to develop strategies to encourage and improve collaboration between the different health experts [30]. These include endocrinologist, obstetrician, midwives, diabetes specialist nurses and dieticians [30]. This will ensure consistency in the health education given to the pregnant women and will in turn improve adherence to therapy.

\section{Facilitators of Adherence to Anti-Diabetic Therapy}

Facilitators of adherence suggested generally addressed the barriers identified. These were subsidization of costs of care, strengthening health education, formal training of health care workers in management of diabetes in pregnancy, collaboration among health care workers and establishment of a unit dedicated to care of pregnant women with diabetes. However the solutions suggested are in- 
terrelated as presented in this section.

\section{* Subsidization of costs of care}

There is need for subsidization of costs of care in diabetes in pregnancy in order to improve access to care and adherence. In some developed countries such as Australia there are schemes such as the National Diabetes Service Scheme (NDSS) that helps in acquisition of essentials such as urine testing and glucose testing machines and strips are subsidized [31].

\section{* Formal training of health care workers in management of diabetes in} pregnancy and strengthening health education

Health professionals play an important role in guiding self-care management of diabetes [32]. They must understand and assess individuals, provide emotional and clinical support, skills and knowledge to patients so that they develop autonomy and be responsible for the control of their disease [32]. There is however, no capacity building in the case of diabetes in general. Healthcare professionals must adjust their knowledge of normal pregnancy to accommodate the additional complexities presented by diabetes [33]. For women with pre-existing diabetes, a successful pregnancy requires planning and expert support [33]. Optimal pregnancy outcomes rely heavily on the quality of diabetes management immediately prior to and during pregnancy [30]. There is need for formal training or in-service training of the professionals to enable them to give relevant health education to patients.

Health educators such as certified health education specialists, certified medical assistants, case managers, lay health and community health workers, and peer counsellors or educators have been contribute effectively as part of the Diabetes Self-Management Education (DSME) team and in providing diabetes self-management support (DSMS) [34]. They may contribute to the provision of DSME instruction and provide DSMS if they have received training in diabetes management, the teaching of self-management skills, group facilitation, and emotional support [34]. These cadres can serve the same purpose in diabetes in pregnancy. In the case of resource limited setting village health workers, community health workers and peer educators can be utilized to complement the efforts of professional health care workers in giving health education.

Collaboration among health care workers and establishment of a unit dedicated to care of pregnant women with diabetes

Management of diabetes in pregnancy requires a clear pathway, developed between all stakeholders, with delineated roles and responsibilities to ensure that best-practice care is delivered along the continuum of antenatal, postnatal, interconception and longer-term care [35]. Delivery of coordinated, effective programs is essential for women with diabetes in pregnancy. Whenever possible, care of pregnant women with diabetes should be given by a multidisciplinary patient-centred team [36]. Women with diabetes in pregnant are at risk of developing perinatal complications that require the development of multidisciplinary programs at centers of excellence that greatly reduce maternal, fetal, and neonatal complications [36]. The centres of excellence suggested are commensurate the 
units dedicated to the care of pregnant women with diabetes suggested in this study

\section{Limitations}

The study had its limitations. The use of purposive sampling compromises the generalizability of the study findings. However sample size was determined by saturation.

\section{Conclusion}

The barriers identified and possible solutions suggested can be employed in developing effective frameworks to promote adherence to anti-diabetic therapy in pregnant women with diabetes. It is important to know the barriers to adherence from the patient's perspective in order to draft holistic solutions to the problem of poor adherence to anti-diabetic therapy in pregnant women with diabetes.

\section{Acknowledgements}

This project was wholly funded by the NORHED Grant to the University Of Zimbabwe College Of Health Sciences, Department of Nursing Science.

\section{References}

[1] Fong, A., Serra, A., Herrero, T., Pan, D. and Ogunyemi, D. (2014) Pre-Gestational versus Gestational Diabetes: A Population Based Study on Clinical and Demographic Differences. Journal of Diabetes and Its Complications, 28, 29-34.

[2] American Diabetes Association (2012) Diagnosis and Classification of Diabetes Mellitus. Diabetes Care, 35, S64-S71. https://doi.org/10.2337/dc12-s064

[3] American Diabetes Association (2015) Management of Diabetes in Pregnancy. Diabetes Care, 38, S77-S79.

[4] International Diabetes Federation (2013) Diabetes Atlas. 6th Edition, International Diabetes Federation, Brussels.

[5] Macaulay, S., Dunger, D.B. and Norris, S.A. (2014) Gestational Diabetes Mellitus in Africa: A Systematic Review. PLoS ONE, 9, e97871. https://doi.org/10.1371/journal.pone.0097871

[6] Zeck, W. and McIntyre, H.D. (2008) Gestational Diabetes in Rural East Africa: A Call to Action. Journal of Women's Health, 17, 403-411. https://doi.org/10.1089/jwh.2007.0380

[7] Hall, V., Thomsen, R.W., Henriksen, O. and Lohse, N. (2011) Diabetes in Sub-Saharan Africa 1999-2011: Epidemiology and Public Health Implications. A Systematic Review. BMC Public Health, 11, 564. https://doi.org/10.1186/1471-2458-11-564

[8] Kuti, M.A., Abbiyesuku, F.M., Akinlade, K.S., et al. (2011) Oral Glucose Tolerance Testing Outcomes among Women at High Risk for Gestational Diabetes Mellitus. Journal of Clinical Pathology, 64, 718-721. https://doi.org/10.1136/jcp.2010.087098

[9] Jesmin, S., Akter, S., Akashi, H., et al. (2014) Screening for Gestational Diabetes Mellitus and Its Prevalence in Bangladesh. Diabetes Research and Clinical Practice, 103, 57-62.

[10] National Institute of Health and Care Excellence (NICE) (2015) Diabetes in Preg- 
nancy: Management from Conception to the Postnatal Period. https://www.nice.org.uk/guidance/ng3/resources

[11] Utz, B., Delamou, A., Belaid, L. and De Brouwere, V. (2016) Detection and Management of Diabetes during Pregnancy in Low Resource Settings: Insights into Past and Present Clinical Practices. Journal of Diabetes Research, 2016, Article ID: 3217098. https://doi.org/10.1155/2016/3217098

[12] Jiwani, A., Marseille, E., Lohse, N., Damm, P., Hod, M. and Kahn, J.G. (2012) Gestational Diabetes Mellitus: Results from a Survey of Country Prevalence and Practices. The Journal of Maternal-Fetal \& Neonatal Medicine, 25, 600-610. https://doi.org/10.3109/14767058.2011.587921

[13] Haque, M., Navsa, M., Emerson, S.H., Dennison, C.R. and Levitt, N.S. (2005) Barriers to Initiating Insulin Therapy in Patients with Type 2 Diabetes Mellitus in Public-Sector Primary Health Care Centres in Cape Town. Journal of Endocrinology, Metabolism and Diabetes of South Africa, 10, 94-99. https://doi.org/10.1080/22201009.2005.10872127

[14] Crowther, C.A., Hiller, J.E., Moss, J.R., McPhee, A.J. and Jeffries, W.S. (2005) Effect of Treatment of Gestational Diabetes Mellitus on Pregnancy Outcomes. The New England Journal of Medicine, 352, 2477-2486.

https://doi.org/10.1056/NEJMoa042973

[15] Bener, A., Saleh, N.M. and Al-Hamaq, A. (2011) Prevalence of Gestational Diabetes and Associated Maternal and Neonatal Complications in a Fast-Developing Community: Global Comparisons. International Journal of Women's Health, 3, 367-373. https://doi.org/10.2147/IJWH.S26094

[16] Bellows, L. and Nichols, K. (2012) Food and Nutrition Series. Diabetes: Nutrition and Health.

[17] American Diabetes Association (2012) Standards of Medical Care in Diabetes. Diabetes Care, 35, S4-S10.

[18] Sui, Z., Grivell, R.M. and Dodd, J.M. (2012) Antenatal Exercise to Improve Outcomes in Overweight or Obese Women: A Systematic Review. Acta Obstetricia et Gynecologica Scandinavica, 91, 538-545.

[19] Gardner, B., Wardle, J., Poston, L. and Croker, H. (2011) Changing Diet and Physical Activity to Reduce Gestational Weight Gain: A Meta-Analysis. Obesity Reviews, 12, e602-e620. https://doi.org/10.1111/j.1467-789X.2011.00884.x

[20] Bosworth, H.B., Eugine, Z.O. and Weinberger, M. (2006) Theoretical Models to Understand Treatment Adherence. In: Patient Treatment Adherence: Concepts, Interventions, and Measurements, Taylor and Francis e-Library.

[21] Shriraam, V., Rani, M.A., Sathiyasekaran, B.W. and Mahadevan, S. (2013) Awareness of Gestational Diabetes Mellitus among Antenatal Women in a Primary Health Center in South India. Indian Journal of Endocrinology and Metabolism, 17, 146148. https://doi.org/10.4103/2230-8210.107861

[22] Mersereau, P., Williams, J.K., Collier, S.A., Mulholland, C., Turay, K. and Prue, C. (2011) Barriers to Managing Diabetes during Pregnancy: The Perceptions of Health Care Practitioners. Birth, 38, 2. https://doi.org/10.1111/j.1523-536X.2010.00464.x

[23] Guest, G., Bunce, A. and Johnson, L. (2006) How Many Interviews Are Enough? An Experiment with Data Saturation and Variability. Field Methods, 18, 59-82. https://doi.org/10.1177/1525822X05279903

[24] Braun, V. and Clarke, V. (2006) Using Thematic Analysis in Psychology. Qualitative Research in Psychology, 3, 77-101. https://doi.org/10.1191/1478088706qp063oa

[25] Miles, M.B. and Huberman, A.M. (1994) Data Management and Analysis Methods. 
2nd Edition, Sage Publications Inc.

[26] Miles, M.B., Huberman, A.M. and Saldaña, J. (2014) Fundamentals of Qualitative Data Analysis. 3rd Edition, Sage, Thousand Oaks, 69-104.

[27] Bell, J. (2015) Reducing Barriers to Glucose Control in Patients with Gestational Diabetes. American Nurse Today, 10, 1-3.

[28] Nielsen, K.K., de Courten, M. and Kapur, A. (2012) Health System and Societal Barriers for Gestational Diabetes Mellitus (GDM) Services-Lessons from World Diabetes Foundation Supported GDM Projects. BMC International Health and Human Rights, 12, 33. https://doi.org/10.1186/1472-698X-12-33

[29] Goldschmidt, V.J. and Colletta, B. (2016) The Challenges of Providing Diabetes Education in Resource-Limited Settings to Women with Diabetes in Pregnancy: Perspectives of an Educator. Diabetes Spectrum, 29, 101-104. https://doi.org/10.2337/diaspect.29.2.101

[30] Singh, H., Murphy, H.R., Hendrieckx, C., Ritterband, L. and Speight, J. (2013) The Challenges and Future Considerations Regarding Pregnancy-Related Outcomes in Women with Pre-Existing Diabetes. Current Diabetes Reports, 13, 869-876. https://doi.org/10.1007/s11892-013-0417-5

[31] Diabetes Australia (2016) Gestational Diabetes Caring for Yourself and Your Baby. https://static.diabetesaustralia.com.au/s/fileassets/diabetes-australia/8f48dbe1-a2b34bb8-8822-0aa6954e8aec.pdf

[32] Torres, H.C., Franco, L.J., Stradioto, M.A., Hortale, V.A. and Schall, V.T. (2010) Evaluation of Group and Individual Strategies in a Diabetes Education Program. Journal of Public Health, 43, 291-298.

[33] Meltzer, S.J. (2005) Management of Diabetes in Pregnancy: Challenges and Trends. Canadian Journal of Diabetes, 29, 246-256.

[34] American Association of Diabetes Educators (AADE) (2015). https://www.diabeteseducator.org/docs/default-source/default-document-library/co mmunity-health-workers-in-diabetes-management-and-prevention.pdf?sfvrsn $=0$

[35] Wilkinson, S.A., Lim, S.S., Upham, S., Pennington, A., O’Reilly, S.L., Asproloupos, D., McIntyre, H.D. and Dunbar, J.A. (2014) Who's Responsible for the Care of Women during and after a Pregnancy Affected by Gestational Diabetes? Medical Journal of Australia, 201, S78-S81. https://doi.org/10.5694/mja14.00251

[36] Kitzmiller, J.L., Block, J.M., Brown, F.M., Catalano, P.M., Conway, D.L., Coustan, D.R., Kirkman, M.S., et al. (2008) Managing Pre-Existing Diabetes for Pregnancy: Summary of Evidence and Consensus Recommendations for Care. Diabetes Care, 31, 1060-1079. https://doi.org/10.2337/dc08-9020 
Submit or recommend next manuscript to SCIRP and we will provide best service for you:

Accepting pre-submission inquiries through Email, Facebook, LinkedIn, Twitter, etc. A wide selection of journals (inclusive of 9 subjects, more than 200 journals)

Providing 24-hour high-quality service

User-friendly online submission system

Fair and swift peer-review system

Efficient typesetting and proofreading procedure

Display of the result of downloads and visits, as well as the number of cited articles Maximum dissemination of your research work

Submit your manuscript at: http://papersubmission.scirp.org/

Or contact jdm@scirp.org 